\title{
COVID-19 Vaccine-Induced Immune Thrombosis with Thrombocytopenia (VITT) and the Shades of Grey in Thrombus Formation
}

\author{
Jecko Thachil, MD, FRC Path ${ }^{1}$ \\ ${ }^{1}$ Department of Haematology, Manchester University Hospitals, \\ Oxford road, Manchester, United Kingdom \\ Semin Thromb Hemost 2022;48:15-18.
}

\author{
Address for correspondence Jecko Thachil, MD, FRC Path, \\ Department of Haematology, Manchester Royal Infirmary, Oxford \\ road, Manchester M13 9WL, United Kingdom \\ (e-mail: jecko.thachil@mft.nhs.uk).
}

Historically, two types of clots have been described in the medical literature-the so called "white clot," where the platelets appear to dominate, and the so called "red clot," where fibrin network with trapped red cells represents the main elements of the thrombi. A vessel-based distinction has also been chronicled where platelet-rich clots occur in arterial thrombosis while activation of coagulation factors is proposed to cause venous thrombosis. ${ }^{1}$ Accordingly, antiplatelet drugs are the treatment of choice where arterial thrombosis occurs, whereas anticoagulants are the typical drug therapy in venous thrombosis. In 2021, vaccine-induced immune thrombocytopenia and thrombosis (VITT) has been proposed to occur as a result of vaccination against COVID-19 (coronavirus disease 2019) with adenovirus-based vaccines. This represents a serious thrombotic disorder where both platelets (evident in thrombocytopenia) and coagulation factors (associated decreased fibrinogen and raised Ddimers) are likely to play a significant role. ${ }^{2-4}$ With both platelets and the clotting system involved, can VITT provide clues to the observed shades of gray (rather than a clear red and white distinction) often seen in thrombus formation? Should we consider more than just antiplatelets or anticoagulants in treating or preventing extension of thrombus formation in the future?

\section{Contribution to Venous Thrombi from Platelets and Gray Zone Triggers}

Rudolf Virchow is suggested to have described the pathophysiology of venous thrombi. ${ }^{5}$ Thrombosis specialists consistently use the eponymous "triad" in their assessment of patients with venous thrombosis. Hypoxia around the venous valves is another trigger for venous thrombosis. ${ }^{6}$ Increased knowledge of immunothrombosis or thromboinflammation (especially since the COVID-19 pandemic) has put the immune system in the limelight to be a contributor to thrombosis. ${ }^{7}$ In all these situations, anticoagulants are used to treat or prevent clot formation, and it is believed there is "little" contribution from platelets. However, more recently, platelets have been suggested to be key participants in unexplained venous thrombotic events. ${ }^{8,9}$ In support of this concept, the Warfarin and Aspirin (WARFASA) and Aspirin to Prevent Recurrent Venous Thromboembolism (ASPIRE) investigators demonstrated approximately $30 \%$ reduction in recurrent venous thrombosis with aspirin compared with placebo. ${ }^{10,11}$ The Antiplatelet Trialists' Collaboration also confirmed a reduction in deep vein thrombosis and pulmonary embolism by using antiplatelet thromboprophylaxis in both surgical and medical patients. $^{12}$ Despite the important role of platelets in the immune and hemostatic response, consideration of antiplatelet agents to treat or prevent venous thrombi in the setting of immunothrombosis and secondary to infections is often an after-thought. Similar to the contribution made by platelets, several other triggers (the immune system, neutrophil extracellular traps, the complement activation, and the contact system-all potentially belonging to the gray zone) may also need to be accounted for in the development of venous thrombosis to prevent anticoagulation failure. But, cogitation for anti-inflammatory agents and drugs which can impact on these triggers to prevent thrombus development is again often a late deliberation.

\section{Contribution to Arterial Thrombi from Activated Coagulation and the Gray Zone Triggers}

Platelets are clearly implicated in causing arterial thrombosis. Aspirin has been used since antiquity (in the form of salicylate-rich plants) and brought a Nobel prize to those who proved its cardioprotective effects. ${ }^{13}$ Since the discovery of its antithrombotic effects, the use of simpler (aspirin) and newer generation antiplatelets has become the cornerstone published online October 8, 2021
Issue Theme Maintaining Hemostasis and Preventing Thrombosis in COVID-19-Part III; Guest Editors: Emmanuel J. Favaloro, PhD, FFSc (RCPA) and Giuseppi Lippi, MD (c) 2021. Thieme. All rights reserved. Thieme Medical Publishers, Inc., 333 Seventh Avenue, 18th Floor, New York, NY 10001, USA
DOI https://doi.org/ 10.1055/s-0041-1735453. ISSN 0094-6176. 
for treatment of arterial ischemia. However, when we look at Bizzozero's original figurative descriptions for platelets' role in thrombosis, it is important to note that he took care to sketch a white cell embedded in the platelet aggregate. ${ }^{14}$ In this respect, the role of monocytes in the development and growth of atherosclerotic plaque is well established, and inflammation has been suggested to play a noteworthy contribution to cardiovascular disorders. ${ }^{15}$ Notwithstanding these white cell offerings, only antiplatelets are prescribed for most patients with arterial occlusive disease. However, several of the forenamed gray zone players may also participate in arterial thrombus formation, especially when these clots develop in the absence of well-known risk factors (e.g., in younger individuals and in the absence of atherosclerosis). As another example, hyper-viscosity secondary to polycythemia or leukocytosis, or abundant plasma cells in plasma cell disorders, can increase arterial thrombotic risk. ${ }^{16}$ Two other groups where the risk of arterial thrombi is increased are the systemic inflammatory disorders (vasculitides) and autoimmune conditions, neither of which respond adequately to antiplatelet agents. ${ }^{16}$ Small strides have been made to consider anticoagulants as adjuncts to antiplatelets in arterial thrombotic disorders. The rivaroxaban in patients with a recent acute coronary syndrome (ATLAS ACS) showed rivaroxaban at $2.5 \mathrm{mg}$ twice daily was able to reduce the rate of major adverse events and stent thrombosis in addition to mortality in the ACS setting compared with placebo. ${ }^{17,18}$ Interestingly, in stark contrast to venous thromboses, research into the gray zone contributions in arterial thrombi has led to identification of ADAMTS-13 (a disintegrin and metalloproteinase with thrombospondin motifs 13) and ultra-large von Willebrand factor multimers in the pathogenesis of thrombotic thrombocytopenia purpura (TTP $)^{19}$ and hemolysis-triggered complement activation in complementopathies; both these groups of disorders require specific treatments (plasma exchange/caplacizumab for TTP and eculizumab for complement disorders) other than antiplatelets to inhibit platelet aggregation and ensuing thrombosis.

\section{The Red, White, and Gray in VITT and Other Conditions}

The cloudiness in identifying the causative factors for thrombus formation is probably best explained by VITT. A clinical presentation of thrombocytopenia/thrombosis or thrombocytopenia without bleeding/thrombosis with abnormal coagulation parameters, especially platelets and D-dimer, and after vaccination may suggest a diagnosis of VITT. ${ }^{20}$ Similar to that described in the case of atypical heparin-induced thrombocytopenia (HIT), platelet activation has been suggested to be a major factor in the pathogenesis of this unusual complication (herein platelet factor 4 itself may be prothrombotic). ${ }^{21,22}$ However, unlike in the cases where antiplatelet agents may "prevent" the thrombotic complication from unwanted platelet stimulation, in the case of VITT, antiplatelets, non-heparin anticoagulation, and immune suppressive measures are recommended but not always successful. ${ }^{3,23}$ The clinical picture with VITT mimics those of catastrophic antiphospholipid syndrome, or rampant disseminated intravascular coagulation, where there may be multisystem thrombosis with high mortality unless early diagnosis and treatment may douse the pathogenetic process. One of the less mentioned clinical syndromes in this context is the entity, "thrombotic storm" which very much imitates the clinical presentation of VITT. ${ }^{24}$ First described by Craig Kitchens in 1998, it typically presents acutely in younger, healthy individuals with two or more arterial or venous thromboemboli following an initiating event. ${ }^{25}$ Like VITT, it may be refractory or has atypical response to standard antithrombotic therapy and exacerbations in case of any treatment interruptions. ${ }^{24,25}$ In these different conditions, where multisystem thrombosis rapidly develops following a nonspecific trigger, the absence of clear pathophysiological explanations for where the problem lies, whether it is in the platelet zone, coagulation zone or indeed the gray zone, or a combination of all three means definitive treatment cannot be established (-Fig. 1).

\section{Two-Layered Platelet Activation in VITT?}

Although, the exact pathophysiological process leading to VITT is yet unclear, what we know so far is evidence for intense platelet activation triggered by adenovirus-based COVID-19 vaccines in this condition. ${ }^{21}$ In this context, the two-layer platelet activation experimentally shown by Stalker et al from the Lawrence Brass laboratory may provide clues to the combination of thrombocytopenia and thrombosis in VITT. ${ }^{26}$ These researchers used confocal imaging, genetically engineered mice, and antiplatelet drugs to show different degrees of platelet activation to produce a hierarchically organized clot. ${ }^{26}$ This layered clot has a dense core of highly activated platelets around which an unstable shell of less-activated platelets is overlaid. The core layer forms a dense clot where the platelet aggregate is likely to be more compact. $^{27}$ During the intense platelet activation, platelet glycoprotein IIb/IIIa (integrin $\alpha$ IIb $\beta 3$ ) binds to fibrinogen and facilitates its intracellular uptake (a possible reason for hypofibrinogenemia). ${ }^{28}$ The outer less-activated platelet layer provides the phospholipid surface for plasma coagulation factors to form a loose clot which envelops the dense fibrin network. The looser fibrin mesh may be more easily degraded by the fibrinolytic system, and thus limit the thrombus enlargement, which would otherwise occlude the vessel. Taking the example of VITT, the platelet activation may be so intense that dense clots are formed in the different circulatory beds and deplete both platelet and fibrinogen in the process, thus leading to thrombocytopenia, hypofibrinogenemia and markedly increased D-dimer values. These clots are unlikely to be stopped by anticoagulation treatments alone unless intense platelet activation is also blocked. The degree of platelet activation may also explain the spectrum of clinical presentations in VITT, where some patients present with mild thrombocytopenia and no thrombosis, while others have full blown syndrome of thrombosis with severe thrombocytopenia. The gray parts here also include the unknown pathways of platelet activation which cause the 


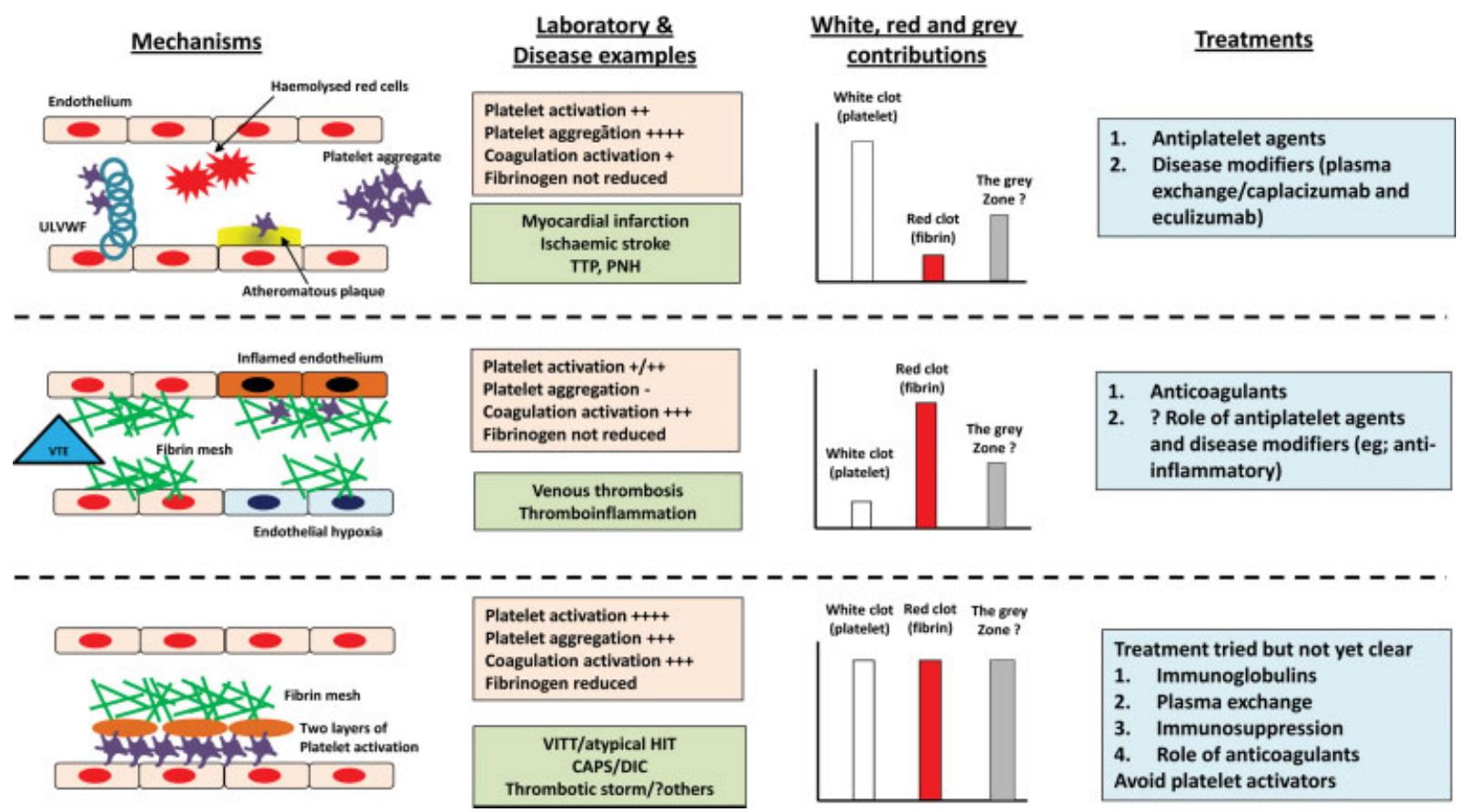

Fig. 1 The red, white and gray shades in thrombus formation. CAPS, catastrophic antiphospholipid syndrome, DIC, disseminated intravascular coagulation; HIT, heparin induced thrombocytopenia; PNH, paroxysmal nocturnal hemoglobinuria; TTP, thrombotic thrombocytopenic purpura.

intense platelet exocytosis which is different to that which occurs in the typical arterial thrombosis.

\section{Looking to the Future}

So, how can we consider the relative contributions of platelets, coagulation activation, and the gray zone factors in the different thrombosis with the currently available laboratory tests (-Fig. 1)?

In a patient presenting with thrombosis:

- Venous normal platelet count and fibrinogen but raised fibrinolytic markers (i.e., D-dimer) suggests coagulation activation predominantly. Anticoagulant may be sufficient. Contributions from platelets and gray contestants need exploration to prevent post-thrombotic complications like post-thrombotic syndrome and pulmonary hypertension.

- Arterial normal platelet count and fibrinogen with no marked elevation of D-dimer suggests platelet activation predominantly. Antiplatelets may be sufficient. Contributions from coagulation factors and gray bodies need exploration to prevent early and late recurrences and management of thrombosis in the absence of atherosclerosis.

- Multi-system-reduced platelet count and fibrinogen and markedly raised D-dimer suggests significant contributions from gray participants in addition to noteworthy subsidies from platelets and activated clotting factors. Although avoiding the trigger factor is the best approach, once the clinical syndrome has developed, multipronged treatment approach is probably necessary to counter the worsening clinical situation.

\section{Conclusion}

In summary, clots are never just red or white. To quote a famous author, there are several "shades of gray" 29 even when it comes to thrombus formation. Scrutinizing alternate pathways to platelet and coagulation factors which can cause thrombosis is important in tailoring patient therapy and decrease the significant morbidity and mortality in those affected. Registry-based studies may help in identifying clinical pointers to the nonplatelet, nonclotting factor contributors to atypical thromboses and early diagnosis in these cases.

Conflict of Interest

None declared.

\section{References}

1 Lippi G, Favaloro EJ. Venous and arterial thromboses: two sides of the same coin? Semin Thromb Hemost 2018;44(03):239-248

2 Oldenburg J, Klamroth R, Langer F, et al. Diagnosis and management of vaccine-related thrombosis following AstraZeneca COVID-19 vaccination: guidance statement from the GTH. Hamostaseologie 2021;41(03):184-189

3 Makris M, Pavord S, Lester W, Scully M, Hunt B. Vaccine-induced immune thrombocytopenia and thrombosis (VITT). Res Pract Thromb Haemost 2021;5(05):e12529

4 Favaloro EJ. Laboratory testing for suspected COVID-19 vaccineinduced (immune) thrombotic thrombocytopenia. Int J Lab Hematol 2021;43(04):559-570 
5 Bagot CN, Arya R. Virchow and his triad: a question of attribution. Br J Haematol 2008;143(02):180-190

6 Bovill EG, van der Vliet A. Venous valvular stasis-associated hypoxia and thrombosis: what is the link? Annu Rev Physiol 2011;73:527-545

7 Gaertner F, Massberg S. Blood coagulation in immunothrombosis -at the frontline of intravascular immunity. Semin Immunol 2016;28(06):561-569

8 Montoro-García S, Schindewolf M, Stanford S, Larsen OH, Thiele T. The role of platelets in venous thromboembolism. Semin Thromb Hemost 2016;42(03):242-251

9 Undas A, Brummel-Ziedins K, Mann KG. Why does aspirin decrease the risk of venous thromboembolism? On old and novel antithrombotic effects of acetyl salicylic acid. J Thromb Haemost 2014;12(11):1776-1787

10 Becattini C, Agnelli G, Schenone A, et al; WARFASA Investigators. Aspirin for preventing the recurrence of venous thromboembolism. N Engl J Med 2012;366(21):1959-1967

11 Brighton TA, Eikelboom JW, Mann K, et al; ASPIRE Investigators. Low-dose aspirin for preventing recurrent venous thromboembolism. N Engl J Med 2012;367(21):1979-1987

12 Antiplatelet Trialists' Collaboration. Collaborative overview of randomised trials of antiplatelet therapy-III: reduction in venous thrombosis and pulmonary embolism by antiplatelet prophylaxis among surgical and medical patients. Antiplatelet Trialists' Collaboration. BMJ 1994;308(6923):235-246

13 Shampo MA, Kyle RA, Steensma DP. John Robert Vane-British pharmacologist and Nobel laureate. Mayo Clin Proc 2013;88(07): e71

14 Mazzarello P, Calligaro AL, Calligaro A. Giulio Bizzozero: a pioneer of cell biology. Nat Rev Mol Cell Biol 2001;2(10):776-781

15 Libby P. Inflammation in atherosclerosis. Arterioscler Thromb Vasc Biol 2012;32(09):2045-2051

16 May JE, Moll S. How I treat unexplained arterial thrombosis. Blood 2020;136(13):1487-1498

17 Connolly SJ, Eikelboom JW, Bosch J, et al; COMPASS investigators. Rivaroxaban with or without aspirin in patients with stable coronary artery disease: an international, randomised, doubleblind, placebo-controlled trial. Lancet 2018;391(10117): 205-218

18 Mega JL, Braunwald E, Wiviott SD, et al; ATLAS ACS 2-TIMI 51 Investigators. Rivaroxaban in patients with a recent acute coronary syndrome. N Engl J Med 2012;366(01):9-19

19 Favaloro EJ, Pasalic L, Henry B, Lippi G. Laboratory testing for ADAMTS13: utility for TTP diagnosis/exclusion and beyond. Am J Hematol 2021;96(08):1049-1055

20 Nazy I, Sachs UJ, Arnold DM, et al. Recommendations for the clinical and laboratory diagnosis of VITT against COVID-19: communication from the ISTH SSC Subcommittee on Platelet Immunology. J Thromb Haemost 2021;19(06):1585-1588

21 Handtke S, Wolff M, Zaninetti C, et al. A flow cytometric assay to detect platelet-activating antibodies in VITT after ChAdOx1 nCov19 vaccination. Blood 2021;137(26):3656-3659

22 Thachil J. The prothrombotic potential of platelet factor 4 . Eur J Intern Med 2010;21(02):79-83

23 Cines DB, Bussel JB. SARS-CoV-2 vaccine-induced immune thrombotic thrombocytopenia. N Engl J Med 2021;384(23): 2254-2256

24 Kitchens CS, Erkan D, Brandão LR, et al. Thrombotic storm revisited: preliminary diagnostic criteria suggested by the thrombotic storm study group. Am J Med 2011;124(04):290-296

25 Kitchens CS. Thrombotic storm: when thrombosis begets thrombosis. Am J Med 1998;104(04):381-385

26 Stalker TJ, Traxler EA, Wu J, et al. Hierarchical organization in the hemostatic response and its relationship to the platelet-signaling network. Blood 2013;121(10):1875-1885

27 Maxwell MJ, Westein E, Nesbitt WS, Giuliano S, Dopheide SM, Jackson SP. Identification of a 2-stage platelet aggregation process mediating shear-dependent thrombus formation. Blood 2007; 109(02):566-576

28 Harrison P, Savidge GF, Cramer EM. The origin and physiological relevance of alpha-granule adhesive proteins. Br J Haematol 1990; 74(02):125-130

29 James EL. Fifty Shades of Grey. London: Vintage Books; 2011 